\title{
CHARACTERIZING THE WHITENESS DEPENDENCE OF THE HERMANN AND SCINTILLATING GRID VISUAL ILLUSIONS
}

\author{
A PREPRINT \\ Eric D. Sun \\ Mather House \\ Harvard University \\ Cambridge, MA 02138 \\ eric_sun@college.harvard.edu
}

June 16, 2019

\begin{abstract}
The Hermann Grid and the Scintillating Grid are among the most prominent brightness-contrast illusions. Perception of these illusions is sensitive to a wide range of image parameters including color, linearity of the edges, and visual size of the images. Here we characterize the influence of three prominent parameters that influence grid illusion perception-dot whiteness, line whiteness, and background whiteness. Experimental data was obtained from several volunteer groups that were tasked with scoring the magnitude of the illusion for images exhibiting different whiteness levels of these three grid elements. Analysis of the data revealed a significant dependence of illusion perception on the whiteness of grid elements. Surprisingly, illusion perception effectively disappeared after an intermediate threshold of whiteness for the dot, line, and background elements in both the Scintillating Grid and Hermann Grid. Moreover, increasing the size of elements decreased the illusion magnitude. The results of this study quantify the whiteness-dependence of brightness-contrast grid illusions and may motivate new experiments to understand the neural mechanisms that are responsible for their perception.
\end{abstract}

Keywords Scintillating Grid $\cdot$ Hermann Grid $\cdot$ visual illusion $\cdot$ contrast $\cdot$ brightness-contrast $\cdot$ whiteness

\section{Introduction}

Visual illusions are key reminders of the limits and vulnerabilities of human perception. For that reason, developing a better understanding of the mechanisms and variables that underlie illusory perception may also improve our understanding of the neural and visual systems. The Hermann Grid (Fig. 1p), which was first discovered in 1870 by Ludimar Hermann [1], is one such prominent visual illusion. The Hermann Grid consists of straight white lines intersecting over a black background and exhibits an illusory perception of dark spots at the grid intersections [2]. The Scintillating Grid, a variant of the Hermann Grid, was discovered in 1994 by E. Lingelbach and consists of white dots at the intersections of gray grid lines on a black background (Fig. 19). The illusion manifests as a fluctuating perception of smaller black dots within the white dots and is sensitive to scanning eye movement [3]. The visual appearance and illusory effect of these two grids are highly similar.

There are several competing theories for the mechanisms and parameters underlying grid illusion perception. An early proposition was that relative differences in lateral inhibition of retinal ganglion receptive fields between the intersections and elsewhere in the grid result in the illusory perception of darker intersection spots in the Hermann Grid [4]. This explanation has been referred to as the retinal ganglion cell theory of Hermann Grid perception [5]. Light is more intense on the inhibitory surround of the receptive fields of on-center neurons at the intersections than at the grid lines, which inhibits lightness perception. On the other hand, the receptive fields of off-center neurons are more activated at the intersection than elsewhere due to more light falling on the activating surround. This relative increment in lateral inhibition is responsible for the perception of dark, illusory spots at the intersections of the Hermann Grid 
[6, 7]. These receptive field inputs may be further propagated by later neural processes, resulting in illusion perception. There is evidence that the Scintillating Grid illusion may also be the product of lateral inhibition, disinhibition, and self-inhibition at early visual processing [8, 9]. Several significant differences exist between experimental observations and the corresponding predictions of the lateral inhibition model for retinal ganglion cell field sizes, field locations, and Herman Grid illusion effects [5]. These differences have led to modified theories where the Scintillating and Hermann Grid illusions manifest later from the simple (S1) cells of the primary visual cortex (V1), perhaps in concert with earlier visual processing [5].

Although the mechanism is still inconclusive, there has been success in identifying modifications that affect the perception of brightness-contrast grid illusions. Perhaps the most important of these have been the contrast and brightness of the Scintillating and Hermann Grids [6, 5]. In addition, modifications in size, distortions in shape, rotation, and undulation of lines in the illusion images can result in qualitative changes in the perception of the illusion and the perceived magnitude of the illusion [5, 10, 11, 12, 13].

Earlier investigations have mostly focused on identifying modifications that enhance or eliminate perception of the grid illusions. However, there is limited work on quantifying the sensitivity of the illusion effect with respect to variations in these modifications. Here we characterize the sensitivity of illusion perception to the variable whiteness levels of individual grid elements in both the Scintillating Grid and Hermann Grid visual illusions. To that end, we recruited several human volunteers to score the magnitude of the illusion effect for varying whiteness levels. From the collected data, statistical analyses and model fitting were performed to identify the range of whiteness values for which illusion perception was present. The fitted models were used to provide additional insight into the mechanistic theories of illusion perception.

\section{a}

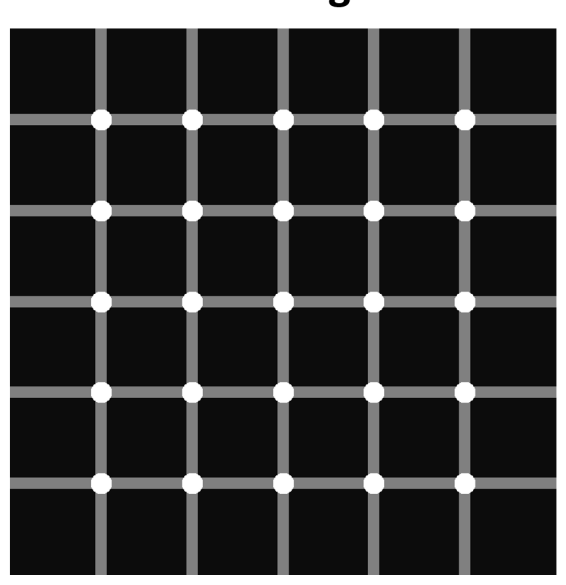

b
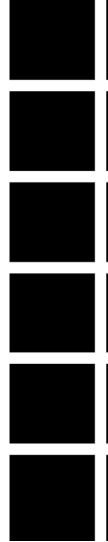

Hermann Grid
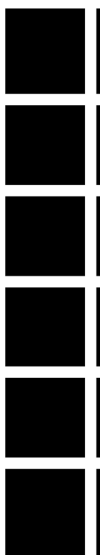

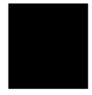

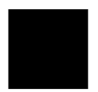

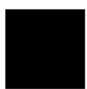

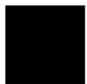

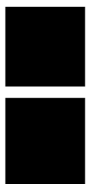

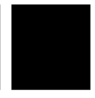

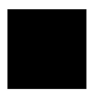

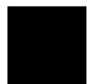

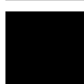
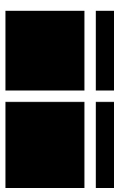
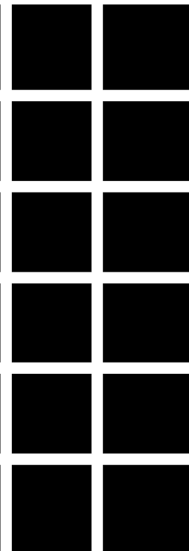

Figure 1: Brightness-Contrast Grid Illusions. (a) The Scintillating Grid illusion presents illusory perception of fluctuating black spots within the white dots. It is defined by dot, line, and background elements. (b) The Hermann Grid illusion presents illusory perception of dark spots at grid intersections. It is defined by line and background elements.

\section{Methods}

\subsection{Perturbation parameter choice}

The most natural division of the Scintillating Grid is into its three constituent elements: dots, lines, background. Since there is evidence that the interplay of these three elements drives illusion perception [3, 2, 11], we expected that modifications to one element could be represented by a combination of similar modifications to the other two elements and vice versa. As a result, similar perturbations may yield complementary effects on illusion perception when applied on different elements of the image. A simpler division could be achieved in the Hermann Grid with its two constituent elements: lines and background. In this investigation, we considered only perturbations in the whiteness level of the pixels that composde each of the grid image elements. 


\subsection{Visual stimuli}

The grid illusion images were computationally generated with dimensions of 768 by 768 pixels. The grid elements and default relative whiteness values of the Scintillating Grid illusion (Fig. 117) were:

- Dots: 25 dots organized in a $5 \times 5$ grid, each of diameter 30 pixels and whiteness $\gamma=1.0$ (white)

- Grid Lines: 5 uniformly spaced lines parallel to the horizon and 5 uniformly spaced lines perpendicular to the horizon. Each line was of width 15 pixels and whiteness $\gamma=0.5$

- Background: whiteness $\gamma=0.0$ (black)

The default settings of the Hermann Grid illusion (Fig. 1p) were:

- Grid Lines: 10 lines organized to intersect evenly, each of width 15 pixels and whiteness $\gamma=1.0$ (white)

- Background: whiteness $\gamma=0.0$ (black)

\subsection{Illusion magnitude}

To evaluate the effect of different whiteness values $\gamma$ on perception of the Scintillating Grid, we introduced the illusion magnitude metric. The illusion magnitude was typically between 0.0 and 1.0 (by steps of size 0.1 ) and was reported by the participants in the study. An illusion magnitude of 0.0 corresponded to no observed illusion whatsoever and the illusion magnitude of 1.0 was set to the baseline level of illusion perception in the original grid illusions (Fig. 1). We expected that perturbations to the whiteness levels of these original images would decrease the illusion magnitude.

\subsection{Periodicity analysis}

The scintillation appearance times were measured as the time in seconds between successive appearances of a black dot within a white dot of the participant's choosing. The participant was instructed to lap a running stopwatch whenever they perceived the illusion in the specified dot. The scintillation appearance times were averaged across all intervals in a ten second period for a given participant to reduce noise. We reported mean and standard deviation values for the time between scintillation events. The Hermann Grid did not produce fluctuating illusion perception and, accordingly, no data was reported for the Hermann Grid stimuli.

\subsection{Experimental setup}

Eight student volunteers were recruited to study the effect of dot whiteness $\gamma$ on illusion perception. They were presented with 21 Scintillating Grid illusion images at different dot whiteness levels ranging from white $(\gamma=1.0)$ to black $(\gamma=0.0)$ by $\Delta \gamma=0.05$ whiteness increments. Participants were positioned one meter away from a 13-inch display screen of luminance 325 candela per square meter situated in a dark room. Under this setup, the grids subtended approximately 12 visual degrees along both axes. Participants were asked to fixate towards the center of the image while maintaining smooth eye movements to induce an illusory perception [10] and to indicate the existence of any illusory effect for each of the 21 images. If an effect was present, the participant was then instructed to report the illusion magnitude corresponding to the perceived illusory effect. The images were presented in descending order of whiteness (i.e. starting with $\gamma=1.0$ and ending with $\gamma=0.0$ ) and there was no time limit for determining illusion presence and assessing the illusion magnitude. As a result, there was no masking between successive images.

In the line whiteness and background whiteness variable experiments, an overlapping cohort of five participants were recruited and exposed to two sets of images. One set varied line whiteness along 21 intervals and the other set varied the background whiteness along the same intervals. In both setups, the other two grid elements were held constant at their default values. Both illusion magnitude and scintillation reappearance times were measured.

In the follow-up investigation with the Hermann Grid, data was collected from a cohort of three participants, all of whom had participated in the previous experiment. Both the background and lines were varied independently along 21 relative whiteness intervals and presented to the participants under the same conditions as those for the Scintillating Grid experiment. Illusion magnitude measurements were collected.

\subsection{Critical point measurement}

We defined the "critical point" $\gamma_{c}$ for a whiteness level experiment as the lowest value of pixel whiteness $\gamma$ (or highest for background whiteness) for which half or more of the participants did not observe any illusion in the image. Since all of the reported relations between the whiteness level of a grid element and illusion perception were monotonic, the 
critical point roughly corresponded to the average whiteness level where a transition occurred between perceiving and not perceiving the illusion effect.

\subsection{Polynomial model fitting}

To estimate the nature of the relation between whiteness and illusion magnitude, we fitted a subset of polynomial functions to the experimental data for nonzero illusion magnitude values. The polynomial model was of the form

$$
y=\alpha\left(x_{c}-x\right)^{\beta},
$$

where $x$ was the variable on the $\mathrm{x}$-axis of the plots and was usually proportional to $\gamma, x_{c}$ was the critical point for $x, \alpha$ was a scaling factor, and $\beta$ was the exponent of the polynomial. A grid search was performed for values of $\alpha \in[0.1, \ldots, 50]$ with $\Delta \alpha=0.198$ and $\beta \in[0.5,1,2]$ and the parameters that minimized the L2 error between the fitted polynomial and the nonzero illusion magnitude values were selected.

All relevant polynomial fitting and period analysis code can be accessed at our public repository: https://github com/sunericd/grid-illusion-whiteness.git

\section{Results}

\subsection{Scintillating Grid Dot Whiteness}

A major difference between the Scintillating and Hermann Grids is the presence of dots at grid intersections. Thus, the dot element is a natural starting point for quantifying the contrast dependence of the Scintillating Grid. Beginning with the original grid (i.e. white dots, $\gamma=1.0$ ), study participants were asked to assign an illusion magnitude to images of decreasing dot whiteness relative to the original grid illusion. That is, an illusion magnitude of $\epsilon$ would correspond to the perception of an illusion that was $\epsilon$ times the illusion effect observed in the first white dot image. On average, as dot whiteness decreased, the illusion magnitude also decreased up until an effective disappearance below $\gamma_{c}=0.60$ (Fig. 2a). This value is the critical point and was determined as the largest dot whiteness value for which at least half of the participants reported perception of the illusion. Further decreasing dot whiteness $\gamma$ past $\gamma_{c}$ did not rescue illusion perception, which implied an approximately monotonic relation between dot whiteness and the strength of the Scintillating Grid illusion. This relationship was best fit with a linear model $(\beta=1)$ specified by $\alpha=1.92$ and minimum L2 error of 0.064. A separate dynamical model with a supercritical Hopf bifurcation at $\gamma=\gamma_{c}$ [14, 15] was also fitted to these experimental observations (details provided in Appendix A).

There was possibly periodic behavior in illusion appearance times among the participants (see Appendix B). Mean estimates of scintillation period for $\gamma=1.0$ and $\gamma=0.5$ were nearly identical, which suggested that there was no dependence of scintillation period on dot whiteness. In order to approximate the frequency $\theta$ of the oscillatory behavior, an average period $(T=1.083 \pm 0.07 \mathrm{~s})$ was calculated from the aggregate period data and the frequency of scintillation ( $f=0.923 \pm 0.056 \mathrm{~s}^{-1}$ ) was calculated as the inverse of the period. The variance between the mean periods of individual participants was $0.25 \mathrm{~s}$.

\subsection{Scintillating Grid Dot Radius}

Although the primary focus of this investigation was to characterize the effect of pixel whiteness on grid illusion perception, a more general question concerns the effect of contrast on the illusion magnitude. In addition to the pixel values, contrast may also depend on factors such as the length of the border between contrasting elements. To investigate the effect of the contrast border length, an overlapping cohort of five participants was presented with variants of the Scintillating with white dots of increasing radius and asked to quantify the illusion magnitude for each image. The perimeter $l$ of the contrast borders scales as $l \sim \tau$ where $\tau$ is the dot radius. The experimental results suggested a critical point for dot radius at $\tau_{c}=1.8$ (equivalent to 26 pixels) (Fig. 2 $2 \mathrm{p}$ ). The majority of the participants perceived a small-radius illusion at $\tau=1.7$ while only one participant perceived the illusion at $\tau=1.8$. At the critical point $\left(\tau_{c}=1.8\right)$, the average illusion magnitude was $2 \% \pm 4.4 \%$ times that of the original dot radius $(\tau=1.0)$ grid. A linear ( $\beta=1$ ) fit with $\alpha=1.11$ was optimal at an L2 error of 0.048 .

There was possibly periodic scintillation in the dot radius experiment. For $\tau=1.3$, estimates of the average scintillation period and frequency were $T=1.347 \pm 0.113 \mathrm{~s}$ and $f=0.742 \pm 0.057 \mathrm{~s}^{-1}$. For $\tau=1.6$, the average period and frequency values were $T=1.815 \pm 0.182 \mathrm{~s}$ and $f=0.551 \pm 0.050 \mathrm{~s}^{-1}$. Inter-participant variances were $0.400 \mathrm{~s}$ and $0.197 \mathrm{~s}$ for $\tau=1.3$ and $\tau=1.6$. Although further work is needed, these preliminary estimates suggest that the frequency of scintillation decreases with increased dot radius. 
a
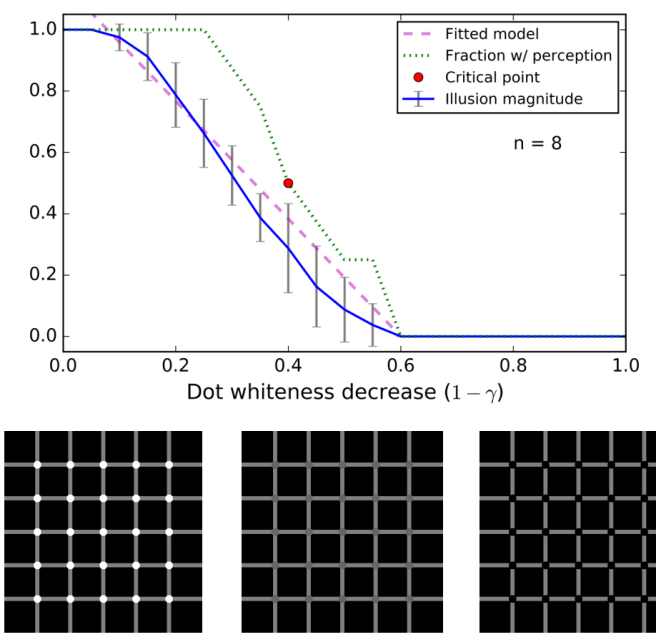

$\gamma=1.0$

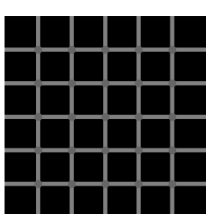

$\gamma=0.60$

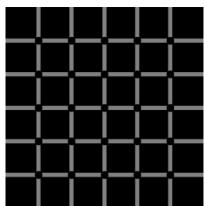

$\gamma=0.0$ b
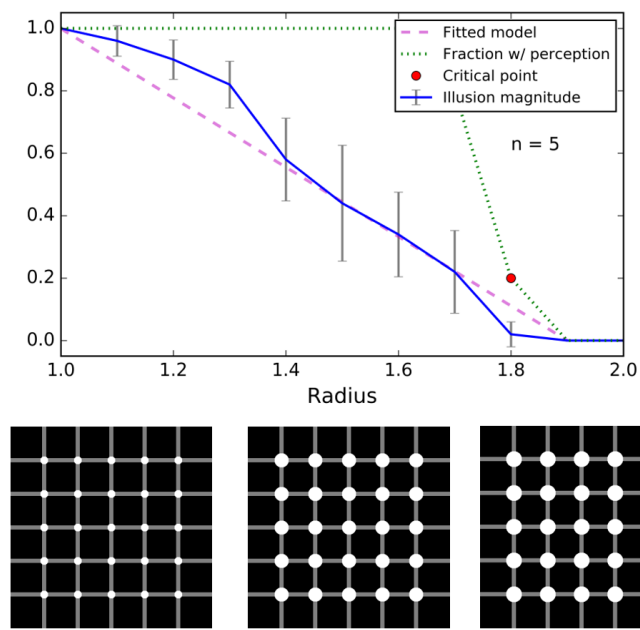

$\tau=1.8$

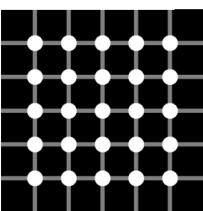

$\tau=2.0$

Figure 2: Scintillating Grid dot whiteness and dot radius. (a) Decreasing the dot whiteness (i.e. $x=1-\gamma$ ) of the Scintillating Grid reduced the magnitude of illusion perception and resulted in a critical point in illusion perception at $\gamma_{c}=0.60$. A linear fit $(\beta=1)$ with $\alpha=1.92$ was optimal with an L2 error of 0.064 . (b) Increasing the radius of the dots similarly decreased the illusion magnitude with a critical point at $\tau_{c}=1.8$. A linear fit with $\alpha=1.11$ was optimal with an error of 0.048 .

\subsection{Scintillating Grid Background Whiteness}

We next investigated the background element using a similar setup as that presented in the dot whiteness experiment. We recruited the same $n=5$ cohort as was used in the dot radius experiment. The whiteness value of the grid background was the variable of interest and was increased from the original value of $\gamma=0.0$ up to $\gamma=1.0$ (i.e a setup that was inverted with respect to the previous dot whiteness experiment). As the background whiteness increased from $\gamma=0.0$ (black) to $\gamma=1.0$ (white), the average illusion magnitude decreased (Fig. 3 a). A critical point around $\gamma_{c}=0.45$ was observed. The relationship was best fit by a linear model with $\alpha=1.92$ and a minimum L2 error of 0.073 .

\subsection{Scintillating Grid Line Whiteness}

The last parameter investigated for the Scintillating Grid was the line element. The first stimuli presented had a grid line whiteness of $\gamma=1.0$ (white) with default values of dot and background whiteness. For these values, the Scintillating Grid effectively reduced to the Hermann Grid illusion. Participants were asked to score the illusion magnitude relative to this image along 21 decreasing intervals of line whiteness until $\gamma=0.0$ (black) was reached (i.e. the setup was identical to the dot whiteness experiment). As line whiteness decreased, the illusion magnitude appeared to decrease in a nonlinear fashion (Fig. 3p) with a critical point at $\gamma_{c}=0.1$. The data was best fit by a square-root $(\beta=0.5)$ model with $\alpha=1.11$ and a L2 error of 0.066 . Notably, this represented the only nonlinear fit that was observed between element whiteness and illusion magnitude across all experiments in this investigation. Furthermore, illusion perception was present for a larger range of line whiteness values as compared to illusion perception for variable dot whiteness and background whiteness, which had more sensitive critical points. These differences may be due to the baseline image for determining illusion magnitude not being the original Scintillating Grid for the line whiteness setup. An estimated frequency of oscillation was calculated as $f=0.763 \pm 0.088 \mathrm{~s}^{-1}$ from a mean period of $T=1.31 \pm 0.17 \mathrm{~s}$.

The notably lower sensitivity of illusion perception to line whiteness suggested that the other two elements (black background and white dots) may exert a greater influence on illusion perception. Since the illusion manifests as black dots scintillating within the white dots, the origin of the illusion may be driven primarily by black and white elements. The simplification of the illusion to solely black and white elements while retaining an illusory effect results in the famous Hermann Grid illusion (Fig. 1p). 
a
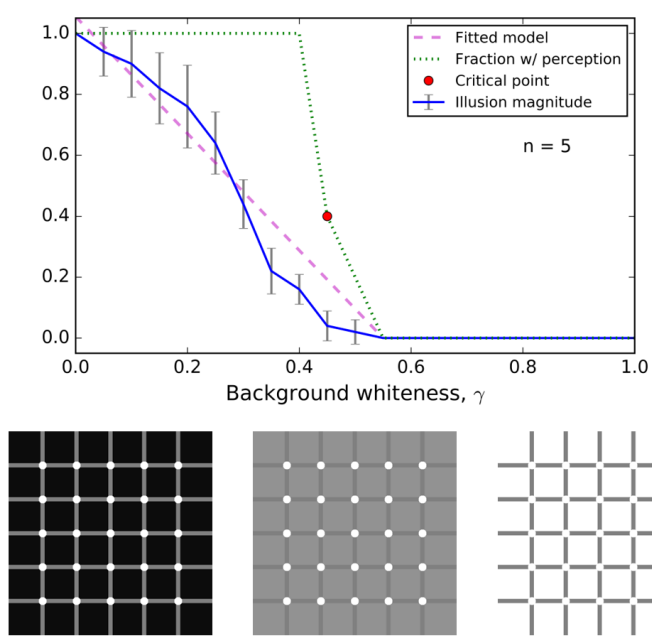

$\gamma=0.0$

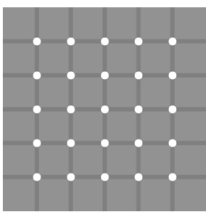

$\gamma=0.45$

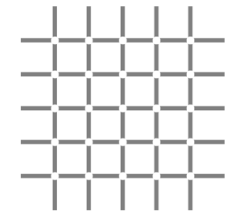

$\gamma=1.0$ b

Decreasing Line Whiteness
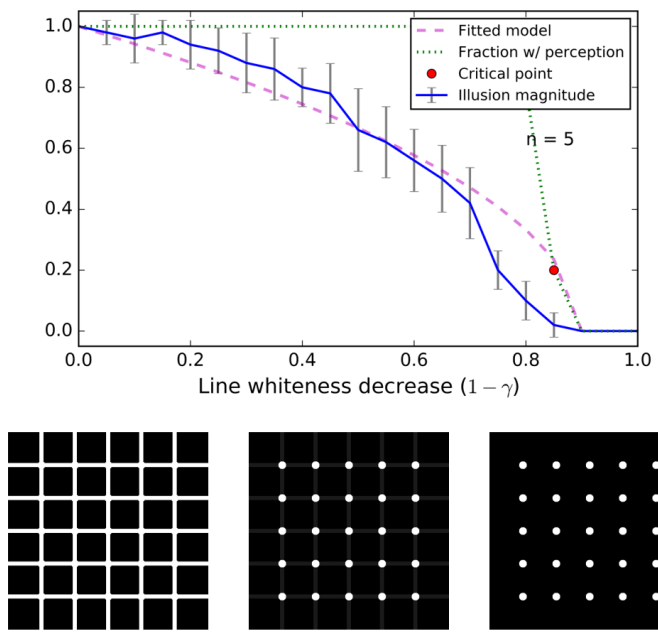

$\gamma=1.0$

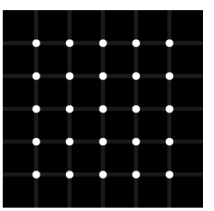

$\gamma=0.15$

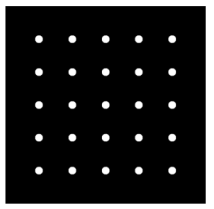

$\gamma=0.0$

Figure 3: Scintillating Grid background whiteness and line whiteness. (a) Increasing background whiteness $(x=\gamma)$ in the Scintillating Grid decreased illusion magnitude and resulted in a perceptual critical point at $\gamma_{c}=0.45$. A linear fit ( $\beta=1$ ) best captured this relationship using $\alpha=1.92$ and a L2 error of 0.073. (b) Decreasing line whiteness in the Scintillating Grid resulted in decreased illusion magnitude and a late critical point at $\gamma_{c}=0.15$. The line whiteness dynamics were most aptly modeled with a quadratic fit ( $\beta=0.5$ ) with scaling of $\alpha=1.11$ and error of 0.066 .

\subsection{Hermann Grid Line Whiteness}

We applied a similar experimental setup to the parent illusion: the Hermann Grid. The Hermann Grid consists of two elements: background and grid lines. Parameterizing the Hermann Grid over line whiteness resulted in decreased illusion magnitude with decreasing line whiteness $\gamma$ (Fig. 4). A critical point was observed around $\gamma_{c}=0.20$. The approach to the critical point was continuous and noticeably linear. The resulting linear fit had a scaling factor of $\alpha=1.11$ and a minimum L2 error at 0.056 .

\section{Hermann Grid Line Whiteness}
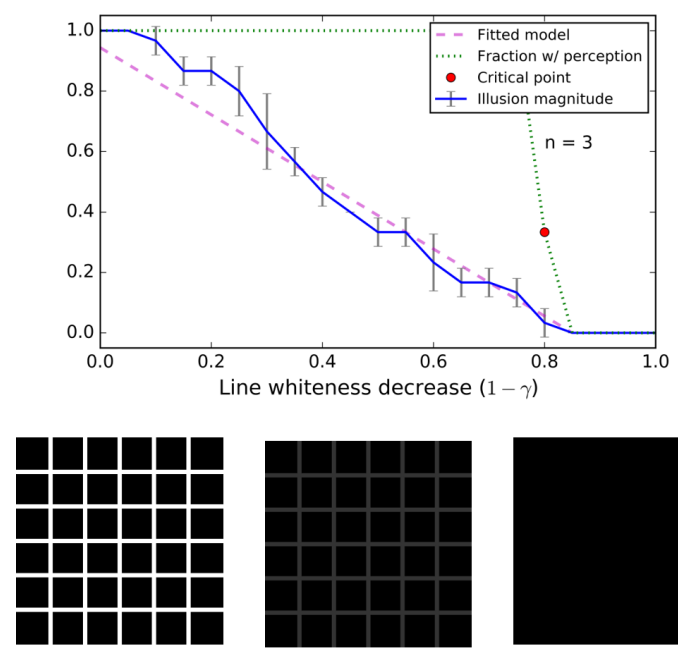

$$
\gamma=1.0
$$

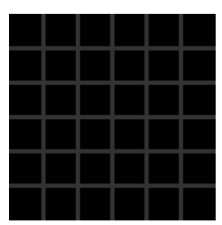

$$
\gamma=0.20
$$

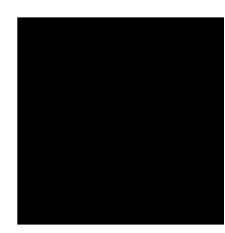

$$
\gamma=0.0
$$

Figure 4: Hermann Grid line whiteness. Decreasing the line whiteness (i.e. $x=1-\gamma$ ) of the Hermann Grid from $\gamma=1.0$ to $\gamma=0.0$ resulted in monotonically decreasing illusion magnitude. A critical point for illusion perception occurred at $\gamma_{c}=0.2$. A linear fit $(\beta=1)$ was optimal with scaling of $\alpha=1.11$ and a total L2 error of 0.056 . 
A PREPRINT - JUNE 16, 2019

\section{Discussion}

\subsection{Main findings}

In this investigation, we characterized the dependence of the Scintillating Grid illusion magnitude on the whiteness pixel values of the dot, line, and background elements, and verified a similar dependence for the Hermann Grid parent illusion. We discovered a monotonic relation between element whiteness and illusion magnitude, and observed a critical threshold for illusion perception at an intermediate whiteness level. Due to contrast, the critical point values of dot, background, and line elements may vary as functions of each other such that the interplay of element whiteness (i.e. contrast) drives illusion perception. In addition, we noted possible periodic behavior for the scintillation of illusory black dots in the Scintillating Grid. Whether this seemingly periodic behavior was a fundamental property of the Scintillating Grid or if it manifested as a result of our experimental setup remains to be determined.

\subsection{Limitations and future directions}

The goal of this investigation was to determine the effect of element contrast on grid illusion perception. As such, non-contrast factors that may affect illusion perception were not investigated. One example is rotation, which weakens Hermann Grid illusion perception and is reflective of perceptual contributions from orientation-specific lateral geniculate and visual cortex cells [2]. Rotation cannot be represented by a combination of element contrasts. Other factors not represented in the experiments or dynamical model (see Appendix A) include variable luminance, size of stimuli projection, and eye movement. There is evidence that global factors such as the order and number of grid lines and intersections may also affect the total illusion magnitude [16]. Other aspects such as chromatic saturation and hue of the grid elements result in similar perceptual effects as contrast [17] and may therefore have similar effects on illusion perception.

It will be of immediate interest to extend the experimental framework to similar illusions. For example, the Ehrenstein whiteness illusion also produces localization of illusory perception at grid intersections [18, 19]. Another promising candidate is White's illusion since it has a similar reliance on element contrast (White, 1979). The Mach Bands, which harbors an illusory perception of gradient from constant colored rectangles, may be another avenue for extending the framework. The Mach Bands, as a low-level brightness-contrast illusion, can be explained relatively well by retinal ganglion cell activity alone [20] much like the Hermann Grid.

\subsection{Theoretical implications}

The results of this investigation corroborate current theories of grid illusion perception. Critical points support the existence of neurological thresholds for illusion perception that are derived from interplay of the ON and OFF regions of receptive fields. The illusion effect disappears if either the OFF or ON regions reaches a critical threshold, which can be achieved by changing the whiteness values or the areas of the grid elements. Therefore, the experimental findings support earlier suggestions that center-surround receptive fields and lateral inhibition constitute a central part of the visual mechanism that underlies illusion perception [4].

Although contrast has been suggested as an important driver of the Hermann Grid illusion [5, 20, 17], our experimental results clarify the relation between element contrast and illusion magnitude as mostly linear. Since the retinal ganglion cell theory posits a direct dependence on the center-surround effect, it predicts a linear dependence of illusion magnitude on dot whiteness $\gamma$ and quadratic dependence on dot radius $\tau$ resulting from second-order changes in area of the dots. The predicted quadratic decline in illusion magnitude due to dot radius is approximate since the spatial effects of some center-surround fields are Gaussian-like rather than constant [21, 22]. Experimentally, however, linear relations to illusion magnitude were observed for both $\gamma$ and $\tau$. It will be necessary to collect data from a larger study cohort in order to confirm these relations. If this difference between the predicted and observed dot radius relations persists, then it would suggest that the lateral inhibition, center-surround theory of a single RGC is untenable. Additional visual processing (i.e. from the V1 or later) may then be necessary to explain the experimentally observed contrast relation [5].

Experimentally, this investigation outlines several exciting properties of the Scintillating Grid: (1) perceptual dependence on contrast, (2) sensitivity to a critical element whiteness threshold which demarcates a region of illusion perception and a region without illusion perception, and (3) possible oscillatory behavior of illusion manifestation. Characterization of other factors influencing Scintillating Grid perception may provide greater insight into the parameter dependence of visual illusions and inform constraints on the neural mechanisms that govern illusion perception. This would be work of broad interest. 
A PREPRINT - JUNE 16, 2019

\section{Acknowledgements}

Many thanks to Dr. Sarah Iams for her helpful conversations regarding the dynamical modeling.

\section{References}

[1] L. Hermann. Eine Erscheinung simultanen Contrastes. Archiv für die gesamte Physiologie des Menschen und der Tiere, 3(1):13-15, December 1870.

[2] Lothar Spillmann. The Hermann Grid Illusion: A Tool for Studying Human Perceptive Field Organization. Perception, 23(6):691-708, June 1994.

[3] MICHAEL Schrauf, Michael, Bernd Lingelbach, and Eugene R Wist. The Scintillating Grid Illusion. Vision Research, 37(8):1033-1038, April 1997.

[4] G. Baumgartner. Indirekte Größenbestimmung der rezeptiven Felder der Retina beim Menschen mittels der Hermannschen Gittertäuschung. Pflüger's Archiv für die gesamte Physiologie des Menschen und der Tiere, 272(1):21-22, January 1960.

[5] Peter H Schiller and Christina E Carvey. The Hermann Grid Illusion Revisited. Perception, 34(11):1375-1397, November 2005.

[6] Lothar Spillmann. Foveal perceptive fields in the human visual system measured with simultaneous contrast in grids and bars. Pflügers Archiv, 326(4):281-299, December 1971.

[7] Eugene R. Wist. Dark adaptation and the Hermann grid illusion. Perception \& Psychophysics, 20(1):10-12, January 1976.

[8] Yingwei Yu and Yoonsuck Choe. A Neural Model of the Scintillating Grid Illusion: Disinhibition and SelfInhibition in Early Vision. Neural Computation, 18(3):521-544, March 2006.

[9] Jacques Ninio and Kent A Stevens. Variations on the Hermann Grid: An Extinction Illusion. Perception, 29(10):1209-1217, October 2000.

[10] Michael Schrauf, Eugene R Wist, and Walter H Ehrenstein. The scintillating grid illusion during smooth pursuit, stimulus motion, and brief exposure in humans. Neuroscience Letters, 284(1):126-128, April 2000.

[11] J. Geier, L. Séra, and L. Bernáth. Stopping the Hermann Grid Illusion by Simple Sine Distortion. In Robert Schwartz, editor, Perception, pages 33-53. Malden Ma: Blackwell, 2004.

[12] Victor de Lafuente and Octavio Ruiz. The orientation dependence of the Hermann grid illusion. Experimental Brain Research, 154(2):255-260, January 2004.

[13] János Geier, László Bernáth, Mariann Hudák, and László Séra. Straightness as the Main Factor of the Hermann Grid Illusion. Perception, 37(5):651-665, May 2008.

[14] Steven Henry Strogatz. Nonlinear Dynamics and Chaos: With Applications to Physics, Biology, Chemistry, and Engineering. Avalon Publishing, 1994. Google-Books-ID: FIYHiBLWCJMC.

[15] J. E. Marsden and M. McCracken. The Hopf Bifurcation and Its Applications. Springer Science \& Business Media, December 2012. Google-Books-ID: FTv0BwAAQBAJ.

[16] Jeremy M Wolfe. Global Factors in the Hermann Grid Illusion. Perception, 13(1):33-40, February 1984.

[17] Andrea McCarter. Chromatic Induction Effects in the Hermann Grid Illusion. Perception, 8(1):105-114, February 1979.

[18] Lothar Spillmann. Perceptual Modification of the Ehrenstein Illusion. In Gestalttheorie in der Modernen Psychologie, pages 210-218. Steinkopff, 1975.

[19] Lothar Spillmann, Kenneth Fuld, and Henk J. M. Gerrits. Brightness contrast in the Ehrenstein illusion. Vision Research, 16(7):713-IN1, January 1976.

[20] K. Ghosh, S. Sarkar, and K. Bhaumik. Low-level brightness-contrast illusions and non classical receptive field of mammalian retina. In Proceedings of 2005 International Conference on Intelligent Sensing and Information Processing, 2005., pages 529-534, January 2005.

[21] Dennis Dacey, Orin S. Packer, Lisa Diller, David Brainard, Beth Peterson, and Barry Lee. Center surround receptive field structure of cone bipolar cells in primate retina. Vision Research, 40(14):1801-1811, June 2000.

[22] Christina Enroth-Cugell and J. G. Robson. The contrast sensitivity of retinal ganglion cells of the cat. The Journal of Physiology, 187(3):517-552, 1966. 
A PREPRINT - JUNE 16, 2019

\section{Appendices}

\section{Appendix A: Dynamical model}

\section{A.1: Dot whiteness model}

A model of dot whiteness in the Scintillating Grid must reproduce the oscillations and decreasing illusion magnitude (represented as the radius of a "illusion-present" circular area with origin at the center of the image). Due to these requirements, polar space served as a natural representation of the grid dynamics. Namely, we defined $r$ to be the illusion effect radius which was proportional to illusion magnitude and $\theta$ to be the angular position of the radius such that $\dot{\theta}$ corresponded with the angular frequency of the illusion's oscillatory behavior. For monotonically decreasing illusion radius until bifurcation with decreasing dot whiteness, we introduced a modified supercritical Hopf bifurcation model, which also permitted oscillatory behavior in the scintillation (i.e $\dot{\theta}$ ) with bifurcation:

$$
\begin{gathered}
\dot{r}=s\left(\gamma-\gamma_{c}\right) r-r^{3} \\
\dot{\theta}=\omega \\
\gamma_{c}=0.60 \quad, \quad \omega=0.923 \pm 0.056 \quad, \quad s \approx 1.8
\end{gathered}
$$

$\gamma_{c}=0.60$ was the dot whiteness bifurcation value, $\omega=0.923$ was the constant oscillation frequency independent of $\gamma$, and $s$ was a scaling factor for fitting to experimental data. $s$ was loosely interpreted as the contrast sensitivity of illusion perception with respect to the dot element. Visualization of the trajectory fields for the fitted model values in $(r, \theta)$ space indicated that we had recovered the desired behavior. When $\gamma>0.60$ (high contrast, high whiteness), all trajectories were attracted to a stable limit cycle which shrunk as $\gamma$ decreased, producing both the radial and oscillatory effects observed experimentally. At $\gamma_{c}=0.60$, a supercritical Hopf bifurcation occurred and the spiral at the origin changed stability through collision with the limit cycle, representing illusion extinction. When $\gamma<0.60$ (low contrast, low whiteness), the trajectories were attracted to the origin $(r=0)$ at comparably faster speed (the origin is a stable spiral), which corresponded to no illusion perception.

\section{A.2: Derivation of the dynamical model}

The radius of the perceived illusion decreased gradually as dot whiteness $(\gamma)$ decreased. A dynamical system with a supercritical Hopf bifurcation in the polar $(r, \theta)$ space would reproduce the characteristic shrinkage and disappearance of the effect radius with decreasing $\gamma$. Conveniently, the Hopf bifurcation exhibits a stable limit cycle that grows with distance from the critical parameter value and disappears for inverse values. The frequency of oscillations, as experimentally confirmed, did not depend on the parameter $\gamma$. We start with the normal form of the supercritical Hopf (Strogatz, 1994; Marsden \& McCracken, 2012) with a single zero-bifurcation parameter $\alpha$ :

$$
\begin{aligned}
& \dot{r}=\alpha r-r^{3} \\
& \dot{\theta}=\phi+b r^{2}
\end{aligned}
$$

Since $\gamma_{c}=0.60$ corresponded to the bifurcation value for dot whiteness $\gamma$, we introduced $\alpha=s\left(\gamma-\gamma_{c}\right)$ where $s$ was an arbitrary scaling factor to fit the experimental data. As required, $\gamma$ solely affected the radius of the illusion. $\phi$ was an arbitrary intercept constant and $b$ was an arbitrary scaling constant to be determined experimentally. $r$ corresponded to the "effect radius" of the illusion and served as a measure of the magnitude of the effect. $\dot{\theta}$, corresponded to the "effect velocity" or the frequency of illusion oscillation. Since there was no evidence that $\dot{\theta}$ depended on the radius $r$ or $\operatorname{dot}$ whiteness $\gamma$, we set $b \approx 0$. Therefore, we simplified the frequency term to $\dot{\theta}=\omega$, which represented a constant experimental frequency. It followed:

$$
\begin{gathered}
\dot{r}=s\left(\gamma-\gamma_{c}\right) r-r^{3} \\
\dot{\theta}=\omega \\
\gamma_{c}=0.60 \quad, \quad \omega=0.923 \pm 0.056 \quad, \quad s \approx 1.8
\end{gathered}
$$

A similar derivation can be achieved for the other supercritical Hopf bifurcation models: Scintillating Grid background whiteness and Hermann Grid line whiteness. 
Appendix B: Possible oscillatory trends in illusion scintillation

a
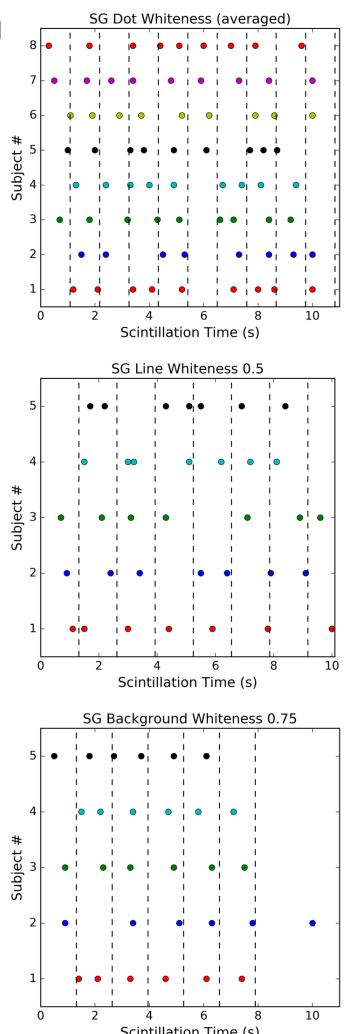

SG Dot radius 1.3
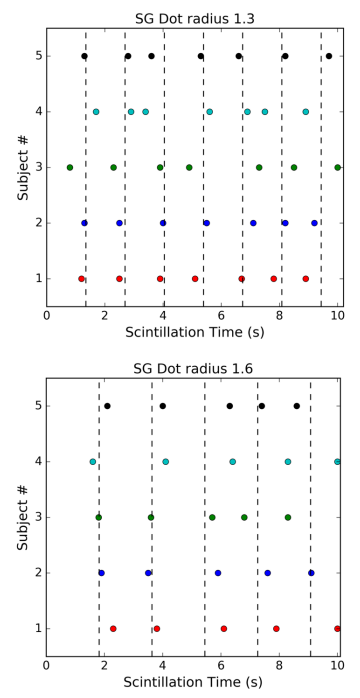
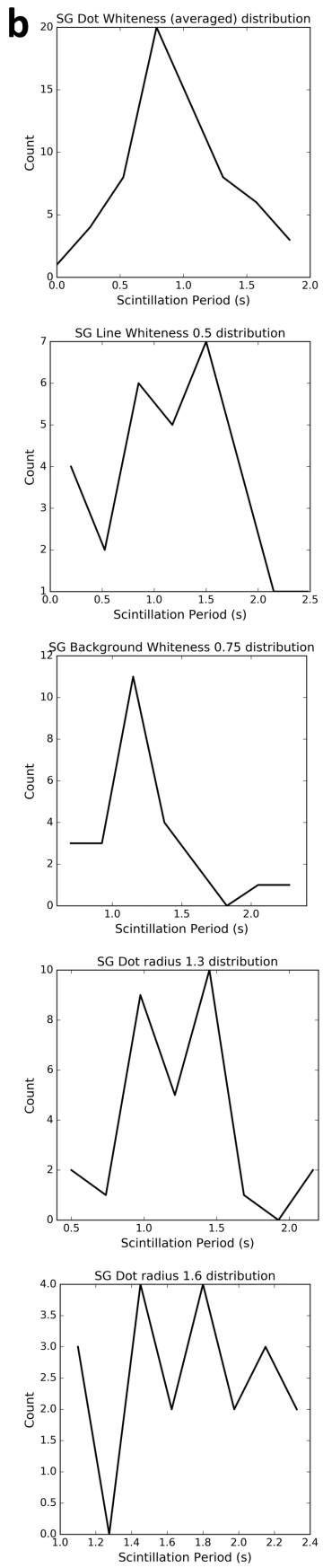

C
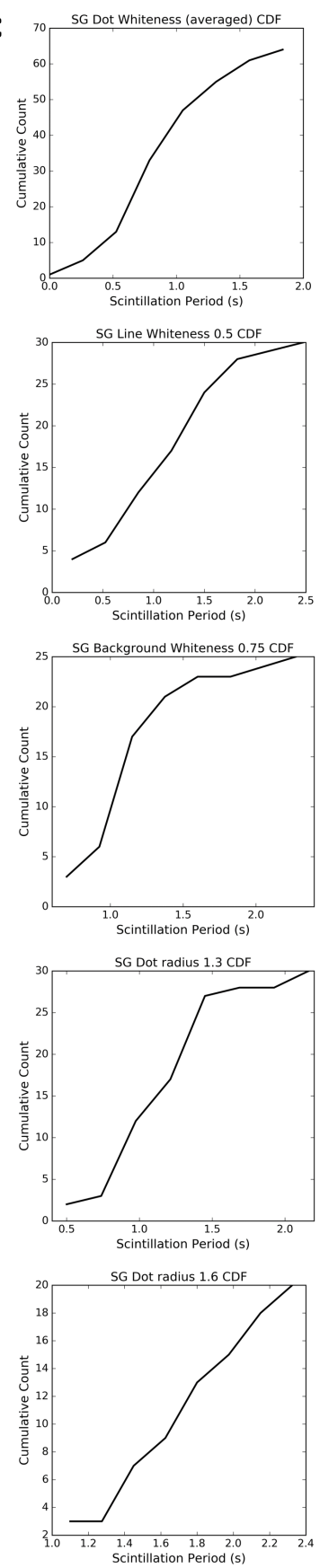

Figure 5: Grid illusions may exhibit regular oscillatory behavior. (a) Illusion appearance (i.e. scintillation) seemed to have regular periodic behavior and low variance in average periods between individuals. This was true across all experimental variations. (b) The distribution of individual periods was approximately Gaussian, which supported a mean period of illusion perception. (c) Cumulative distributions of the scintillation times also appeared to have an inflection point, which was indicative of a mean period of oscillation. It should, however, be noted that the observed oscillatory action may be an artifact of the experimental instruction for participants to maintain smooth eye movements, and further work is needed to determine the significance of these trends. 